\title{
TOLERANCE TO ALUMINIUM TOXICITY IN TANZANIAN SORGHUM GENOTYPES
}

\author{
J.H. RINGO, E.E MNENEY, A.O. ONKWARE ${ }^{1}$, B.A. WERE ${ }^{1}$, E.J. TOO ${ }^{1}$, J.O. OWUOCHE ${ }^{2}$ \\ and S.O. GUDU ${ }^{1}$ \\ Mikocheni Agricultural Research Institute, P. O. Box 6226, Dar Es Salaam, Tanzania \\ ${ }^{1}$ Department of Biological Sciences, Moi University, P. O. Box 1125-30100, Eldoret, Kenya \\ ${ }^{2}$ Department of Biotechnology, Moi University, P. O. Box 1125-30100, Eldoret, Kenya \\ Corresponding author: ringojustinh@yahoo.com
}

\begin{abstract}
Aluminium (Al) toxicity is a major abiotic constraint on grain sorghum (Sorghum bicolor L. Moench) production on acid soils in East Africa. Aluminium in acidic soil inhibits water and mineral uptake from and consequently, reduces plant vigour and yield. A study was done to determine genetic diversity of Tanzania's sorghum for response to Al toxicity. Five day old seedlings of 98 sorghum genotypes were subjected to 0,148 or 222.25 moles of $\mathrm{Al}^{3+}$ supplied as Al2 (SO4)3.16H2O in Hoagland's nutrient solution. Seedlings were raised in a growth chamber for five days, after which root lengths were recorded. Net root growth was used to discriminate the germplasm into phenotypic groups. The genotype MCSR T33 exhibited highest net root length and was classified as tolerant. Wahi, MCSR T69 and MCSR T11 were moderately tolerant, while the rest were susceptible.
\end{abstract}

Key Words: Genetic diversity, root length, Sorghum bicolor

\section{RÉSUMÉ}

La toxicité aluminique est une contrainte majeur à la production du sorhgo (Sorghum bicolor L. Moench) sur les sols acides en Afrique de l'est. L'aluminium (Al) des sols acides inhibe l'assimilation d'eau et de minéraux du sol, et réduit par conséquent la vigueur des plantes et le rendement.Une étude était faite pour déterminer la diversité génétique du sorgho de la Tanzanie en réponse à la toxicité aluminique. Les plants agés de 5 jours issus de 98 génotypes de sorgho étaient soumis à 0,148 ou 222.25 moles de $\mathrm{Al}^{3+}$ fournis sous forme de $\mathrm{Al} 2$ (SO4)3.16H2O dans une solution de nutriment de Hoagland. Les plantules étaient plantées dans la chambre de croissance pendant 5 jours après lesquels la longueur des racines était mesurée. La croissance nette des racines était utilisée pour séparer les racines en groupes phénotypiques. Le génotype MCSR T33 avait exhibé une longueur nette plus élevée des racines et était classifié comme tolérant. Wahi, MCSR T69 et MCSR T11 étaient modérément tolérant, alors que les restes étaient susceptibles.

Mots Cles: Diversité génétique, longueur des raciness, Sorghum bicolor

\section{INTRODUCTION}

Sorghum (Sorghum bicolor L. Moench) is one of the important staple cereals in the semi-arid regions of the world (Rohrbach et al., 2002). It is an important food and feed crop, and is becoming an industrial crop used in biofuels and brewing. It is an appropriate crop for cultivation in semi-arid lands of Eastern Africa because of its relative tolerance to drought. Moreover, it performs better under low soil fertility than the other locally grown cereals. It has been identified as a crop that can improve livelihoods of the vulnerable communities (World Bank, 2005) living in the arid environments. However, lack of superior cultivars arising from limited 
research on sorghum improvement in the region, coupled with drought and acidity often result in low yields (800 $\mathrm{kg}^{-1}$ compared to 2-3.0 $\mathrm{t} \mathrm{ha}^{-1}$ world average) (INTSORMIL/ USAID, 2006).

Aluminium toxicity often occurs in acidic soils and is one of major abiotic stresses that limit sorghum productivity worldwide (Magalhaes et al., 2004). Moreover, over 40\% of the arable lands are acidic (Von Uexkull and Mutert, 1995). Most sorghum production in East Africa occurs on soils with $\mathrm{pH}<5.5$. In fact, in some parts, $\mathrm{Al}$ saturation is high (4-55\%) and dramatically affects the availability of phosphorus (Kanyanjua et al., 2002). Acid soils cover more than $15 \%$ of the agricultural land in Tanzania (MARI, 2006), and over 7.5\% in Kenya of arable land (Kanyanjua et al., 2002).

Previous studies indicate that Al tolerance in plants is largely influenced by a putatively orthologous series of at least two major loci that are inherited as major $\mathrm{Al}$ tolerance genes in sorghum and wheat (Magalhaes et al., 2007). Tolerance to $\mathrm{Al}$ in sorghum is controlled by a major gene $A l t_{S B}$, located on chromosome 3 (Magalhães et al., 2004). The quantitative trait locus (QTL) located on chromosome 1 of rice is orthologous to the Alt $t_{S B}$ sorghum gene, while the QTL found on chromosome 3 of rice is orthologous to the $A l t_{B H}$ wheat genes (chromosome 4DL) and to barley Alp on chromosome 4H (Magalhães et al., 2004). The ALMT1 gene, which encodes a malate transporter activated by Al, was cloned by Sasaki et al. (2004); and was found to be related to $\mathrm{Al}$ tolerance in wheat. In rice (Oryza sativa), Al tolerance is a quantitative trait and QTL studies identified $\mathrm{Al}$ tolerance loci in all the 12 rice chromosomes (Nguyen et al., 2003).

Several techniques have been developed for more rapid evaluation of tolerance to soil acidity. Among those is bioassay that includes nutrient solutions (Duncan et al., 1983; Magnavaca et al., 1987). Screening sorghum genotypes for tolerance to $\mathrm{Al}$ toxicity has been done through $\mathrm{Al}$-induced root growth inhibition (Magalhaes et al., 2004), callose production and Al-content in root tips in nutrient solution (Baligar et al., 1989). Solution culture is cheap, fast and the most commonly used method in $\mathrm{Al}$ toxicity screening experiments. It provides easy access to root systems, tight control over nutrient availability and $\mathrm{pH}$, and non-destructive measurement of tolerance (Carver and Ownby, 1995). It has been applied for $\mathrm{Al}$ tolerance analysis in alfalfa (Baligar et al., 1989), cowpea (Paliwal et al., 1994); barley (Ma et al., 1997); maize (Conaado et al., 1999); tomato and rape (Luo et al., 1999); Soybean (Villarcia et al., 2001) and in sorghum (Magalhaes et al., 2004). The inhibition of seminal root growth by $\mathrm{Al}$ in the nutrient solution is used to quantify $\mathrm{Al}$ tolerance in crops. Magnavaca et al. (1987) developed an extensively applied protocol that uses basal nutrient solution for screening for $\mathrm{Al}$ tolerance. Root length measurement is the most suitable criterion for $\mathrm{Al}$ stress is studies in maize and sorghum. It is also suitable for identifying genotypes with superior alleles for Al tolerance (Hede et al., 2002).

This study was done to identify new sources of $\mathrm{Al}$ tolerance in sorghum and determine the level of variation for tolerance to $\mathrm{Al}$ toxicity in the Tanzanian sorghum germplasm.

\section{MATERIALS AND METHODS}

Sorghum accessions used in the study. Ninety eight sorghum accessions were collected from sorghum growing areas in Tanzania (Table 1). Five commercial released varieties (Hakika, Macia, Pato, Tegemeo and Wahi) were obtained from Ilonga Research Centre in Morogoro $\left(9^{\circ}\right.$ 4' 0 " S and 36 51' 0 " E) in Tanzania. Sorghum standards for $\mathrm{Al}$ tolerance were obtained from International Crops Research Institute for the Semi-Arid (ICRISAT).

The study materials were screened for $\mathrm{Al}$ tolerance using nutrient solution as the growth media according to procedure described by Magnavaca et al. (1987). Seedlings were subjected to $\mathrm{Al}$ treatments of 0 (control), 148, $222 \mu \mathrm{M}$ supplied as AlK $\left(\mathrm{SO}_{4}\right)_{2} \cdot 16 \mathrm{H}_{2} \mathrm{O}$. Sorghum seeds were surface sterilised in $1 \%$ sodium hypochlorite $(\mathrm{NaOCl})$ for 8 minutes and then rinsed through 8 times using sterile distilled water. Seeds were then germinated between moistened sterilized $20 \mathrm{~cm} \times 20 \mathrm{~cm}$ Velvex ${ }^{\circledR}$ paper towels in an incubator at $25^{\circ} \mathrm{C}$ in the dark for 3 days. Initial root length (irl) was measured before the seedling were put in growth 
Tolerance to aluminium toxicity in sorghum

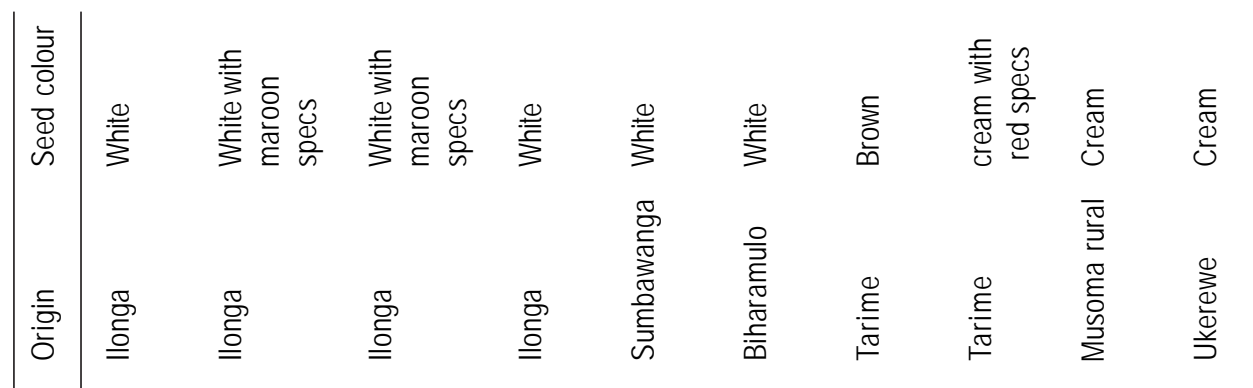

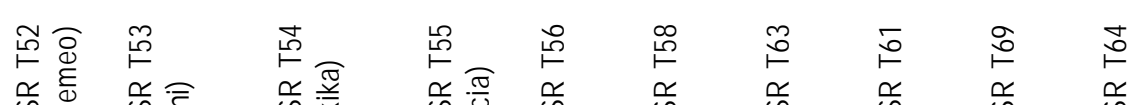

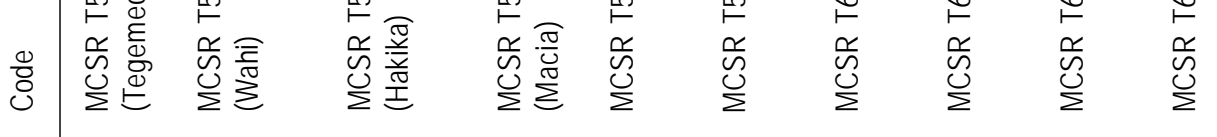

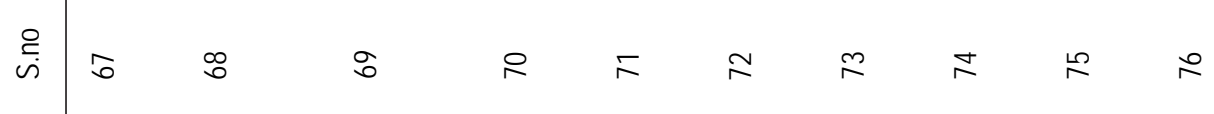

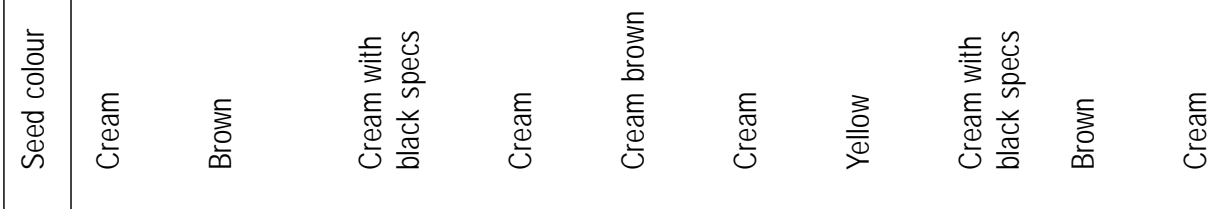

(g)In

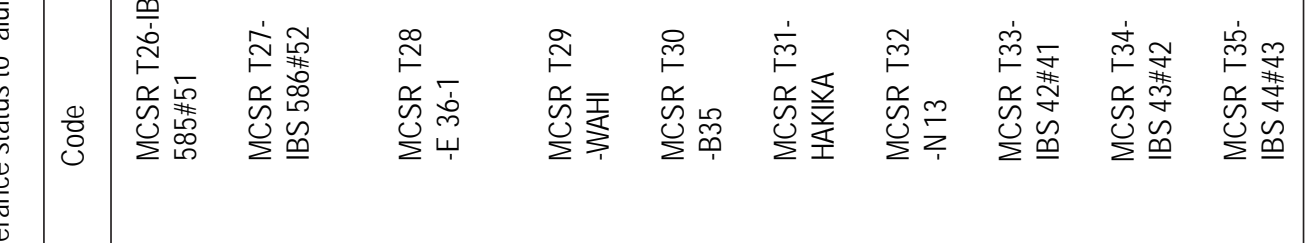

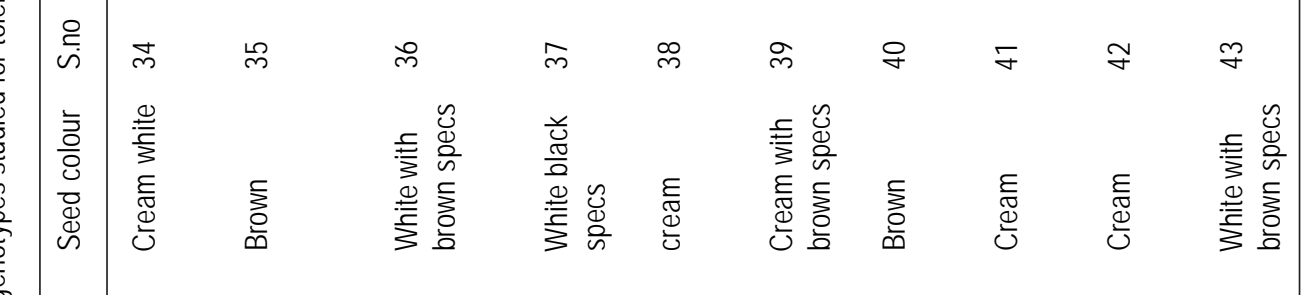

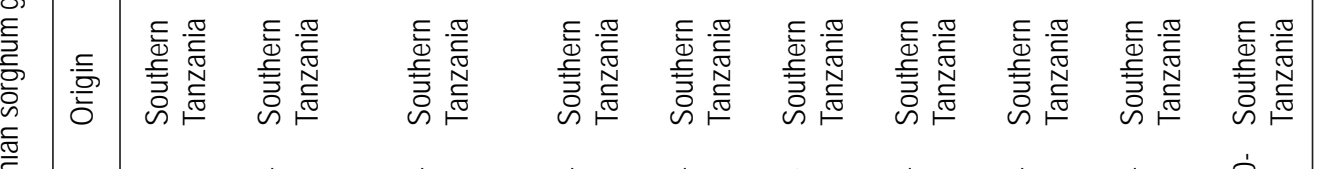

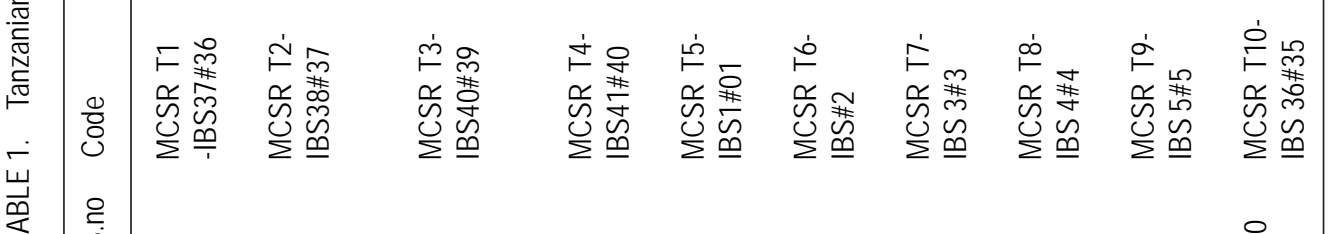


J.H. RINGO et al.

\begin{tabular}{|c|c|c|c|c|c|c|c|c|c|c|c|}
\hline $\begin{array}{l}\frac{\bar{z}}{8} \\
\frac{8}{8} \\
\bar{c} \\
心\end{array}$ & $\begin{array}{l}\frac{\xi}{0} \\
\frac{0}{0} \\
\frac{1}{8} \\
0\end{array}$ & $\frac{\mathscr{g}}{\xi}$ & 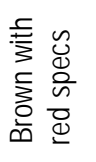 & 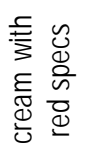 & $\begin{array}{l}\frac{5}{0} \\
\frac{0}{8} \\
\frac{1}{8} \\
0\end{array}$ & $\stackrel{0}{\frac{0}{\xi}}$ & $\begin{array}{l}\text { క్థ్ } \\
\stackrel{0}{0}\end{array}$ & $\frac{0}{3}$ & $\frac{0}{3}$ & $\stackrel{\frac{0}{\xi}}{\xi}$ & 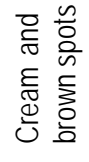 \\
\hline t) & 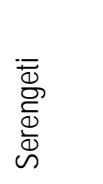 & 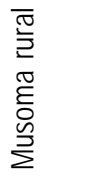 & 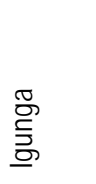 & $\begin{array}{l}\text { on } \\
\stackrel{5}{0}\end{array}$ & 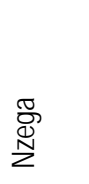 & 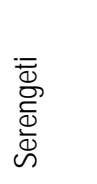 & 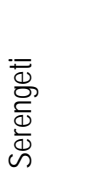 & $\begin{array}{l}\frac{0}{0} \\
\frac{0}{d} \\
\text { J }\end{array}$ & 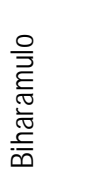 & $\begin{array}{l}\sum_{0}^{0} \\
\frac{d}{2} \\
\text { J }\end{array}$ & 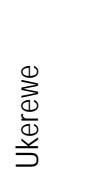 \\
\hline $\begin{array}{l}\frac{1}{8} \\
\\
\end{array}$ & $\begin{array}{l}\hat{\rho} \\
\frac{r}{0} \\
0 \\
\Sigma\end{array}$ & 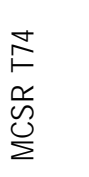 & $\begin{array}{l}\frac{5}{2} \\
\frac{0}{0} \\
0 \\
\Sigma\end{array}$ & $\begin{array}{l}\overrightarrow{9} \\
\vec{r} \\
\vec{v} \\
\dot{0}\end{array}$ & 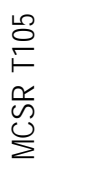 & $\begin{array}{l}\stackrel{\infty}{\kappa} \\
\frac{r}{0} \\
\frac{\delta}{2}\end{array}$ & $\begin{array}{l}\stackrel{m}{\infty} \\
= \\
\frac{r}{0} \\
0 \\
\Sigma\end{array}$ & 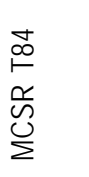 & $\begin{array}{l}E \\
\frac{r}{\sqrt{3}} \\
\sum\end{array}$ & $\begin{array}{l}\frac{10}{F} \\
\frac{\alpha}{0} \\
0 \\
\Sigma\end{array}$ & $\begin{array}{l}\overrightarrow{1} \\
\infty \\
\frac{\alpha}{0} \\
\delta \\
\Sigma\end{array}$ \\
\hline $\begin{array}{l}\frac{q}{\omega} \\
\dot{\omega})\end{array}$ & $\kappa$ & $\stackrel{\infty}{\wedge}$ & $\stackrel{9}{R}$ & ه & $\vec{\infty}$ & $\infty$ & $\cong$ & $\Phi$ & 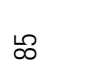 & $\stackrel{\infty}{\infty}$ & $\infty$ \\
\hline $\begin{array}{l}\bar{z} \\
\frac{\bar{z}}{8} \\
\bar{z} \\
\mathrm{~d} \\
\omega\end{array}$ & 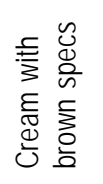 & 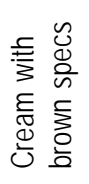 & 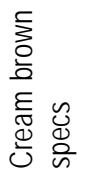 & 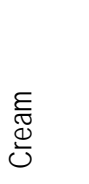 & 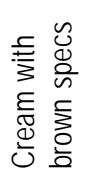 & 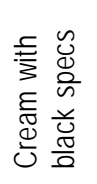 & 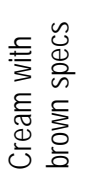 & 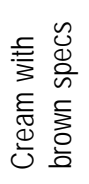 & 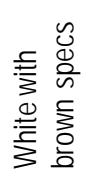 & 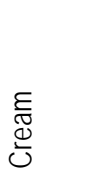 & 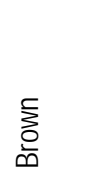 \\
\hline के & 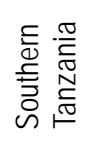 & 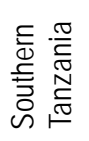 & 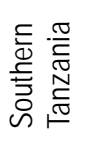 & 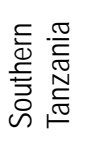 & 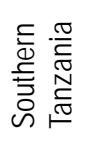 & 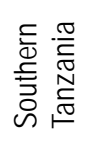 & 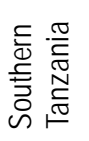 & 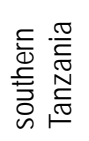 & 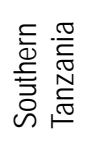 & 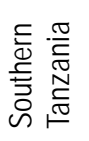 & 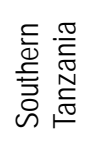 \\
\hline$\frac{1}{8}$ & 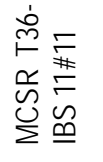 & 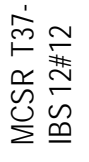 & 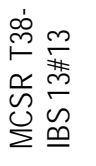 & 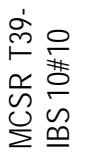 & 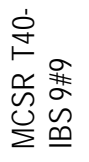 & 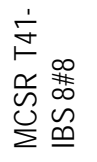 & 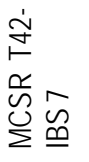 & 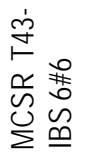 & 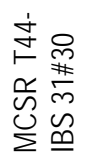 & 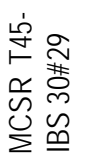 & 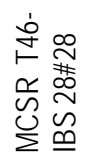 \\
\hline$\frac{?}{q^{\circ}}$ & $\forall$ & ஜf & $q$ & $f$ & $\stackrel{\infty}{q}$ & g & 8 & பે & กี & חె & 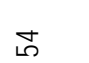 \\
\hline $\begin{array}{l}\bar{z} \\
\bar{z} \\
\overline{8} \\
\overline{8} \\
0\end{array}$ & 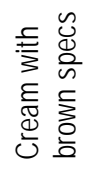 & 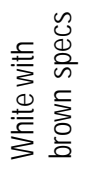 & $\frac{\mathscr{m}}{\xi}$ & $\sum_{\bar{\emptyset}}^{\xi}$ & 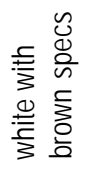 & 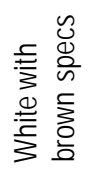 & 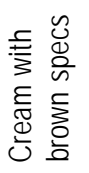 & $\begin{array}{l}\tilde{\Phi} \\
\stackrel{\varpi}{\tilde{\sigma}}\end{array}$ & 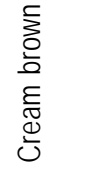 & $\begin{array}{l}\tilde{\Xi} \\
\underset{\tilde{\sigma}}{0}\end{array}$ & 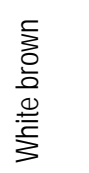 \\
\hline 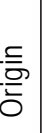 & 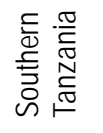 & 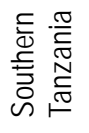 & 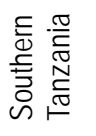 & 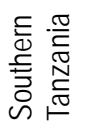 & 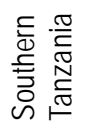 & 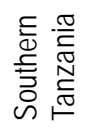 & 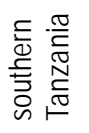 & 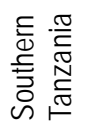 & 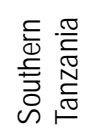 & 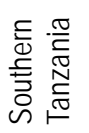 & 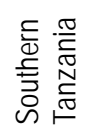 \\
\hline 8 & 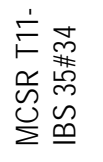 & 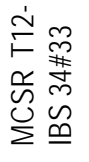 & 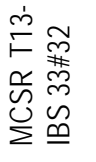 & 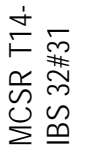 & 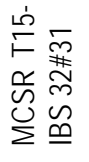 & 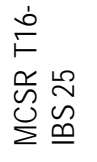 & 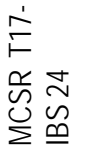 & 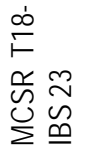 & 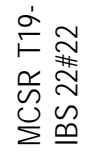 & 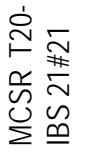 & 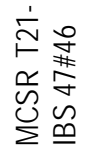 \\
\hline & 7 & ๆ & $\stackrel{\mathcal{M}}{\mathcal{G}}$ & A & & $\stackrel{\oplus}{\varphi}$ & ને & $\stackrel{\infty}{\rightarrow}$ & 구 & ㅇ & \\
\hline
\end{tabular}


Tolerance to aluminium toxicity in sorghum

\begin{tabular}{|c|c|c|c|c|c|c|c|c|c|}
\hline $\begin{array}{l}\bar{z} \\
\overline{0} \\
\frac{8}{0} \\
\$ \\
0\end{array}$ & 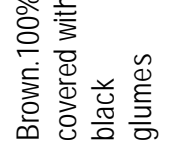 & 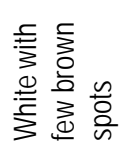 & 畗 & $\sum_{\overline{0}}^{\mathfrak{\Phi}}$ & $\stackrel{0}{\frac{0}{3}}$ & $\frac{\mathscr{g}}{\xi}$ & 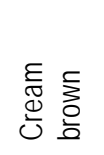 & $\sum_{\overline{0}}^{\mathfrak{\Phi}}$ & $\stackrel{0}{\xi}$ \\
\hline : & 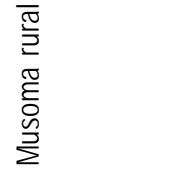 & 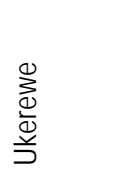 & 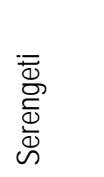 & 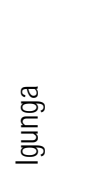 & 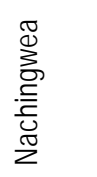 & 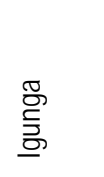 & & & \\
\hline$\frac{0}{8}$ & $\begin{array}{l}\tilde{F} \\
\frac{r}{0} \\
\frac{0}{2}\end{array}$ & $\begin{array}{l}\infty \\
\infty \\
\frac{1}{0} \\
\dot{0} \\
\sum\end{array}$ & $\begin{array}{l}\infty \\
\stackrel{0}{\bullet} \\
\frac{r}{0} \\
0 \\
\Sigma\end{array}$ & $\begin{array}{l}8 \\
8 \\
1 \\
\frac{\pi}{0} \\
\sum\end{array}$ & $\begin{array}{l}\stackrel{8}{9} \\
\frac{1}{0} \\
0 \\
\Sigma\end{array}$ & $\begin{array}{l}\overrightarrow{0} \\
\stackrel{1}{\circ} \\
\frac{r}{y} \\
\dot{y}\end{array}$ & $\begin{array}{l}\hat{2} \\
\frac{r}{0} \\
\frac{0}{2}\end{array}$ & $\begin{array}{l}\infty \\
\infty \\
\frac{1}{0} \\
\frac{0}{2} \\
\sum\end{array}$ & $\begin{array}{l}8 \\
9 \\
\frac{1}{0} \\
0 \\
\sum\end{array}$ \\
\hline$\frac{q}{v}$ & $\infty$ & ळ & 8 & б- & ช & ஜ & চ & ஜூ & $\mathscr{8}$ \\
\hline \begin{tabular}{l}
$\bar{z}$ \\
$\frac{0}{8}$ \\
$\frac{8}{8}$ \\
\hdashline
\end{tabular} & 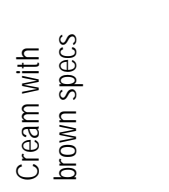 & $\underset{\hat{D}}{\tilde{\omega}}$ & 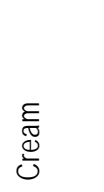 & 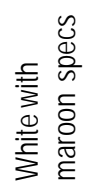 & 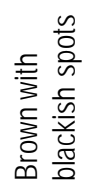 & 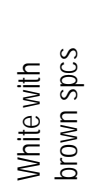 & 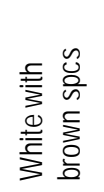 & $\sum_{0}^{\mathfrak{0}}$ & $\stackrel{9}{3}$ \\
\hline 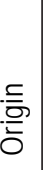 & 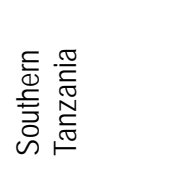 & 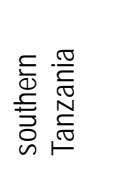 & 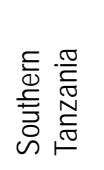 & $\begin{array}{l}\stackrel{\sigma}{\sigma} \\
\stackrel{\sigma}{=}\end{array}$ & 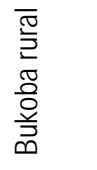 & 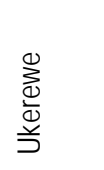 & 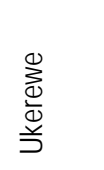 & 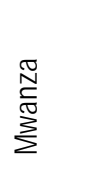 & 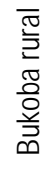 \\
\hline$\frac{0}{8}$ & 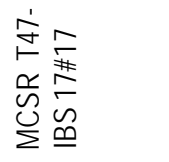 & 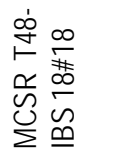 & 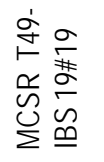 & 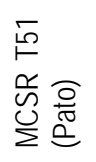 & $\begin{array}{l}8 \\
6 \\
\frac{1}{0} \\
0 \\
\sum\end{array}$ & $\begin{array}{l}\qquad 0 \\
\stackrel{0}{0} \\
\frac{1}{0} \\
0 \\
\sum\end{array}$ & $\begin{array}{l}q \\
\frac{v}{0} \\
\dot{0} \\
\Sigma\end{array}$ & $\begin{array}{l}\stackrel{n}{1} \\
\frac{r}{0} \\
\sum\end{array}$ & $\begin{array}{l}5 \\
E \\
\frac{r}{3} \\
\Sigma\end{array}$ \\
\hline$\frac{\mathscr{q}}{\dot{v}}$ & 绐 & i̊ & $\hat{n}$ & $\stackrel{\infty}{\circ)}$ & ำ & 8 & ర્ઢ & $\widetilde{\sigma}$ & $\widetilde{8}$ \\
\hline 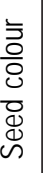 & 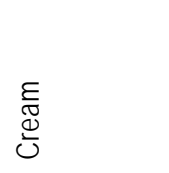 & 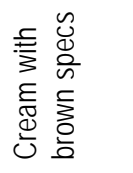 & 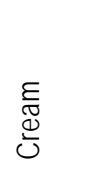 & 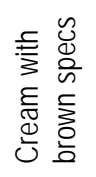 & 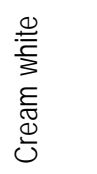 & $\begin{array}{l}\tilde{\Phi} \\
\stackrel{\varpi}{0} \\
\tilde{\sigma}\end{array}$ & $\begin{array}{ll} & y \\
0 & 0 \\
\frac{0}{0} & 0 \\
0 & \vdots \\
0 & \vdots \\
\xi & 0\end{array}$ & 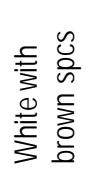 & $\frac{\frac{c}{\omega}}{\sum_{0}^{5}}$ \\
\hline 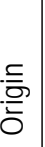 & 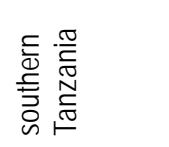 & 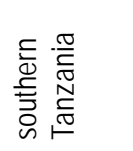 & 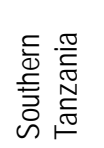 & 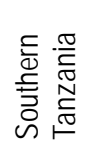 & 焉 & $\begin{array}{l}\frac{0}{3} \\
\frac{0}{00} \\
\frac{0}{J}\end{array}$ & 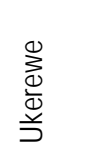 & $\begin{array}{l}\frac{0}{\bar{z}} \\
\frac{\widetilde{\pi}}{\sigma} \\
\frac{\tilde{\omega}}{\bar{\omega}}\end{array}$ & $\begin{array}{l}\text { 또 } \\
\text { 홍 }\end{array}$ \\
\hline$\frac{0}{8}$ & 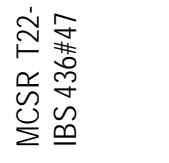 & 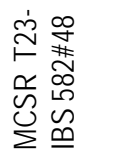 & 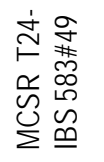 & 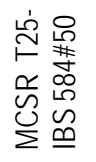 & $\begin{array}{l}\tilde{N} \\
\infty \\
\frac{r}{0} \\
0 \\
\sum\end{array}$ & $\begin{array}{l}0 \\
\infty \\
0 \\
\tilde{y} \\
\Sigma\end{array}$ & $\begin{array}{l}\infty \\
\infty \\
\infty \\
\tilde{n} \\
0 \\
\sum\end{array}$ & $\begin{array}{l}\hat{\infty} \\
\frac{r}{0} \\
0 \\
\sum\end{array}$ & $\begin{array}{l}\text { N } \\
\stackrel{9}{1} \\
\frac{r}{03} \\
0 \\
\Sigma\end{array}$ \\
\hline$\frac{q}{v}$ & N & $\stackrel{m}{N}$ & d & $\stackrel{\llcorner}{N}$ & $\stackrel{\leftrightarrow}{N}$ & $\hat{N}$ & $\stackrel{\infty}{N}$ & ㄱ. & 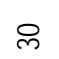 \\
\hline
\end{tabular}


plastic cups ( $2.5 \mathrm{~cm}$ x $3.5 \mathrm{~cm}$ ). Loaded cups were placed on $32.5 \mathrm{~cm} \times 32.5 \mathrm{~cm}$ plastic rafts and transferred to trays containing 8-litre nutrient solutions. The seedlings were raised in a growth chamber with continuous aeration of the nutrient solution aeration pump (FIMA ${ }^{\circledR}$ air compressor) for 5 days at a $\mathrm{pH}$ of 4.2 .

Temperature and light were maintained at 26 ${ }^{\circ} \mathrm{C}$ and $550 \mu \mathrm{mol}$ photons per square metre per second, respectively. Final root length $(f r l)$ was measured from the root tip to the base on the $5^{\text {th }}$ day after transfer to nutrient solution. The net root length (nrl) was used to group sorghum into tolerant and sensitive phenotypic classes.

Data were subjected to analysis of variance and means separated by Least Significant Difference at 5\% probability level using SAS Version 8 (SAS, 2002).

\section{RESULTS}

Overall, final root length and net root length differed significantly $(\mathrm{P}<0.05)$ with $\mathrm{Al}$ concentration (Table 2). The highest root reduction was observed at $222 \mu \mathrm{M} \mathrm{Al}$ treatment and plants grown in this (highest) $\mathrm{Al}$ concentration had stunted roots with blackish tips, typical symptoms of $\mathrm{Al}$ on the meristematic region. This treatment was too severe even for the cultivars that appeared to tolerate the stress imposed by $148 \mu \mathrm{M} \mathrm{Al}$.

Genotypic differences in $\mathrm{Al}$ tolerance among the screened sorghum germplasm was very clear from the fact that the root growth of the genotypes screened in the solution culture varied. Based on net root growth $(n r l)$, MCSR T33 had $n r l$ of 1.94 and was above the $\mathrm{Al}$ tolerant standard check. The standard check, ISCR 110 had $n r l$ of $1.70 \mathrm{~cm}$. Three sorghum genotypes, MCSR T69, T53 and

TABLE 2. Root growth means across treatments of sorghum accessions studied

\begin{tabular}{lccc}
\hline $\begin{array}{l}\text { Al treatment } \\
(\mathrm{mM})\end{array}$ & $\begin{array}{l}\text { Initial root } \\
\text { length }(\mathrm{cm})\end{array}$ & $\begin{array}{l}\text { Final root } \\
\text { length }(\mathrm{cm})\end{array}$ & $\begin{array}{l}\text { Net root } \\
\text { length }\end{array}$ \\
\hline 0 & 3.29 & 7.49 & 4.20 \\
148 & 3.33 & 5.38 & 2.04 \\
222 & 3.28 & 3.78 & 1.50 \\
LSD $(0.05)$ & 0.07 & 0.09 & 0.08 \\
\hline
\end{tabular}


Tolerance to aluminium toxicity in sorghum

TABLE 3. Net root lengths, relative root lengths and Al tolerance status of some genotypes screened for Al tolerance

\begin{tabular}{|c|c|c|c|c|c|c|c|}
\hline Genotype & $\begin{array}{l}\text { Net root } \\
\text { length } \\
0 \mu \mathrm{M}(\mathrm{cm})\end{array}$ & $\begin{array}{l}\text { Net root } \\
\text { length } 148 \\
\mu \mathrm{M}(\mathrm{cm})\end{array}$ & $\begin{array}{l}\text { Aluminium } \\
\text { tolerance } \\
\text { status }\end{array}$ & Genotype & $\begin{array}{l}\text { Net root } \\
\text { length } \\
0 \mu \mathrm{M}(\mathrm{cm})\end{array}$ & $\begin{array}{l}\text { Net root } \\
\text { length } 148 \\
\mu \mathrm{M}(\mathrm{cm})\end{array}$ & $\begin{array}{l}\text { Aluminium } \\
\text { tolerance } \\
\text { status }\end{array}$ \\
\hline T33 & 4.10 & $1.94^{*}$ & $T$ & T66 & 1.58 & 0.49 & $S$ \\
\hline T53 & 5.60 & $1.64^{*}$ & MT & T85 & 3.79 & 0.47 & S \\
\hline ICSR 110 & 2.50 & $1.70^{*}$ & $T$ & $\mathrm{~T} 21$ & 1.44 & 0.46 & $S$ \\
\hline T69 & 4.21 & $1.32^{\star}$ & MT & T54 & 3.26 & 0.44 & $\mathrm{~S}$ \\
\hline T11 & 5.04 & $1.19 *$ & MT & T42 & 4.45 & 0.44 & $S$ \\
\hline T76 & 3.17 & $0.96^{*}$ & $S$ & $\mathrm{~T} 7$ & 2.53 & 0.44 & $S$ \\
\hline T30 & 5.33 & $0.95^{\star}$ & S & T65 & 4.79 & 0.44 & $\mathrm{~S}$ \\
\hline T31 & 3.91 & $0.93^{*}$ & $S$ & T103 & 4.13 & 0.42 & $\mathrm{~S}$ \\
\hline T45 & 2.44 & 0.92 & $S$ & T52 & 3.36 & 0.42 & $S$ \\
\hline T59 & 4.96 & 0.92 & S & T90 & 4.26 & 0.39 & S \\
\hline T3 & 3.31 & 0.91 & $S$ & T79 & 3.78 & 0.39 & $\mathrm{~S}$ \\
\hline T51 & 5.28 & 0.90 & $S$ & $\mathrm{~T} 78$ & 2.77 & 0.38 & $S$ \\
\hline T56 & 1.06 & 0.89 & $S$ & T84 & 3.86 & 0.37 & $\mathrm{~S}$ \\
\hline T41 & 4.09 & 0.87 & $S$ & T27 & 0.41 & 0.36 & $S$ \\
\hline T97 & 2.71 & 0.84 & $S$ & T102 & 3.90 & 0.36 & $S$ \\
\hline $\mathrm{T} 70$ & 1.45 & 0.83 & $S$ & $\mathrm{~T} 4$ & 4.27 & 0.35 & $S$ \\
\hline T19 & 2.91 & 0.83 & S & T36 & 3.03 & 0.35 & $\mathrm{~S}$ \\
\hline T5 & 0.81 & 0.81 & $S$ & T68 & 2.60 & 0.35 & $S$ \\
\hline T38 & 2.59 & 0.81 & S & T16 & 3.87 & 0.32 & $S$ \\
\hline T75 & 0.55 & 0.79 & $S$ & T28 & 3.09 & 0.32 & $S$ \\
\hline T61 & 0.72 & 0.79 & $S$ & T13 & 1.00 & 0.32 & $S$ \\
\hline T96 & 3.25 & 0.77 & S & T29 & 3.44 & 0.30 & $S$ \\
\hline T18 & 4.44 & 0.74 & $S$ & T17 & 2.98 & 0.30 & $\mathrm{~S}$ \\
\hline T43 & 1.76 & 0.72 & $S$ & T82 & 1.63 & 0.30 & $\mathrm{~S}$ \\
\hline T35 & 0.49 & 0.72 & S & $\mathrm{T} 73$ & 2.85 & 0.27 & S \\
\hline T87 & 3.01 & 0.71 & $S$ & T10 & 1.29 & 0.27 & $S$ \\
\hline T25 & 2.09 & 0.71 & $S$ & $\mathrm{~T} 77$ & 0.07 & 0.24 & $S$ \\
\hline T94 & 1.79 & 0.69 & S & T62 & 2.16 & 0.23 & $\mathrm{~S}$ \\
\hline T93 & 1.10 & 0.69 & $S$ & T89 & 4.04 & 0.21 & $S$ \\
\hline Т8 & 3.09 & 0.67 & $S$ & T39 & 3.73 & 0.20 & $\mathrm{~S}$ \\
\hline T64 & 2.48 & 0.67 & S & $\mathrm{T} 72$ & 0.79 & 0.20 & S \\
\hline T74 & 0.94 & 0.66 & $S$ & T63 & 3.01 & 0.19 & $S$ \\
\hline T100 & 3.79 & 0.64 & $\mathrm{~S}$ & T37 & 0.65 & 0.19 & $\mathrm{~S}$ \\
\hline T34 & 3.15 & 0.60 & $\mathrm{~S}$ & T15 & 1.44 & 0.19 & $\mathrm{~S}$ \\
\hline T55 & 4.11 & 0.60 & $S$ & T92 & 1.13 & 0.19 & $S$ \\
\hline T91 & 2.19 & 0.60 & $S$ & T67 & 4.32 & 0.18 & $S$ \\
\hline T81 & 2.76 & 0.57 & S & T47 & 4.78 & 0.18 & $S$ \\
\hline T12 & 1.86 & 0.57 & $S$ & T80 & 4.14 & 0.16 & $S$ \\
\hline T14 & 0.95 & 0.57 & $\mathrm{~S}$ & T26 & 0.73 & 0.15 & $\mathrm{~S}$ \\
\hline T9 & 2.81 & 0.53 & $\mathrm{~S}$ & T40 & 0.71 & 0.15 & $\mathrm{~S}$ \\
\hline T46 & 2.98 & 0.52 & $S$ & T106 & 2.34 & 0.14 & $S$ \\
\hline T23 & 2.80 & 0.52 & $S$ & T71 & 3.42 & 0.12 & $S$ \\
\hline T22 & 1.32 & 0.52 & $S$ & T24 & 2.47 & 0.09 & $S$ \\
\hline T49 & 3.44 & 0.51 & $S$ & $\mathrm{~T} 1$ & 4.97 & 0.08 & $S$ \\
\hline T57 & 4.12 & 0.51 & $\mathrm{~S}$ & T58 & 1.39 & 0.03 & $\mathrm{~S}$ \\
\hline T98 & 2.55 & 0.50 & $S$ & T60 & 0.97 & 0.01 & $\mathrm{~S}$ \\
\hline
\end{tabular}

* significant at $\mathrm{P}<0.05 ; \mathrm{T}=$ tolerant; $\mathrm{MT}=$ medium tolerant; $\mathrm{S}=$ sensitive to Al toxicity. - Scale for classification ( $n r l 148$ $\mu M$ ): T > 1.70cm; MT 1.5- $1.69 \mathrm{~cm} ; \mathrm{S}<1.5 \mathrm{~cm}$ - ICSR 110 was used as standard check from ICRISAT for Al tolerance 
MCSR T 11 were closer to the standard check. On the basis of the same parameter $(n r l)$, sorghum genotypes were grouped into three different classes that is tolerant, medium tolerant and sensitive (Table 3).

\section{DISCUSSION}

Although sorghum root growth was impaired by the presence of $\mathrm{Al}$ in the nutrient solution, there was differential response of genotypes to $\mathrm{Al}$ stress (Table 2). Normally, the root is the plant organ most affected by Al toxicity, and more specifically the root tip is considered to be the main site for Al toxicity (Archambault et al., 1997). As a result, root elongation is considered to be the most sensitive parameter under shortterm exposure to $\mathrm{Al}$ and, therefore, may represent the whole-plant reaction to $\mathrm{Al}$. The inhibition of root elongation seems to explain the retardation in plant growth through reduced nutrient and water uptake, consequently resulting in poor yield. The variability in $\mathrm{Al}$ tolerance has previously been noted in sorghum (Magalhaes et al., 2006), barley (Tamas et al., 2006) and maize (Ligeyo, 2007). This experiment based on net root length to discriminate the genotypes into respective tolerance groups.

The distinct difference in root growths at different levels of aluminium concentration in the nutrient solution indicates that after exposing sorghum roots to aluminum treatments for 5 days, the nutrients uptake by the seedlings was limited due to effect of aluminum on the tips. Root tips are directly involved in nutrients and water absorption by plants. The tolerant genotypes showed little effect of aluminium across the treatments and had better growth.

It was also found that $148 \mu \mathrm{M} \mathrm{Al}$ concentration was sufficient to discriminate tolerant Tanzanian sorghum genotypes from sensitive ones. However, Al concentration at $222 \mu \mathrm{M}$ was too high and this classified tolerant genotypes into sensitive. Majority of the Tanzanian genotypes screened in this study were sensitive to Al stress. Majority of sorghum growing areas are reported to have soils with $\mathrm{pH}$ ranging from 4.5 to 5.5 (MARI, 2006). Therefore, cultivation of the broad germplasm largely aluminium sensitive could be one of the contributing factors to low sorghum production. This justifies the need to breed and select for sorghum cultivar(s) tolerant to Al stress.

Several experiments for selection of genotypes tolerant to $\mathrm{Al}$ in the nutrient solution have been successfully conducted in sorghum (Furlani and Clark, 1981; Giaveno et al., 2001). Galvez and Clark (1991) demonstrated that two sorghum genotypes maintained their relative differences to Al toxicity tolerance independently whether they were grown separately or in the same nutrient solution. According to Magalhaes et al. (2006), genetic variation for Al tolerance in plants has allowed the development of cultivars that are high yielding on acidic, Al toxic soils.

Only one accession MCSR T33 of the sorghum genotypes screened for Al tolerance was classified as tolerant. This genotype had relatively higher net root growth in aluminium treatment as compared to the standard check (Table 2). MCSR T33 was collected from the southern Tanzania. The most sensitive genotype, MCSR T60 was collected from Musoma rural in the Mara region of Tanzania. Three genotypes were in medium tolerant class, while the remaining genotypes were sensitive to $\mathrm{Al}$ toxicity. The medium tolerant genotype T53 (Wahi) is at the same time a Striga tolerant variety (Mbwaga, 2006) which make it a suitable candidate to be included in breeding programmes for developing a multiple stress varieties of sorghum.

\section{ACKNOWLEDGEMENT}

The authors thank Swedish Government (SIDA) through BIO-EARN Project for funding this work. Dr. Mary Mgonja of ICRISAT provided sorghum standard materials.

\section{REFERENCES}

Archambault, D.J., Zhang, G.C. and Taylor, G.J. 1997. Spatial variation in the kinetics of aluminium (Al) uptake in roots of wheat (Triticum aestivum L.) exhibiting differential resistance to $\mathrm{Al}$ - Evidence for 
metabolism-dependent exclusion of Al. Journal of Plant Physiology 151: 668 674.

Baligar, V.C., Elgin, J.H. and Foy, C.D. 1989. Variability in alfalfa for growth and mineral uptake and efficiency ratios under aluminium stress. Agronomy Journal. 81: 223 - 229.

Carver, B. F. and Ownby, J. D, 1995. Acid soil tolerance in wheat. Advanced Agronomy Journal 54: 117-173.

Conaado, G.M.A, Luguercio, L.L,, Martins, P.R., Parentoni, S.N., Paiva, E., Borem, A. and Lopes, M.A. 1995. Hematoxylin staining as a phenotypic index for aluminium tolerance selection in tropical maize. Theorerical and Applied Genetics 99: 747 - 754.

Chantereau, J. and Nicou, R, 1994. Sorghum. The tropical Agricultural series. CTA Wageningen, Netherlands; Mackmillan, London, UK.

Duncan, R.R., Clark, R.B. and Furlani, P.R. 1983. Laboratory and field evaluations of sorghum for response to aluminum and acid soil. Agronomy Journal 75:1023 - 1026.

Furlani, R.R., Clark, R.B1981. Screening Sorghum for aluminium tolerance in nutrient solution. Agronomy Journal 73 587-594.

Galvez, L., Clark, R.B. 1991. Nitrate and ammonium uptake and solution $\mathrm{pH}$ changes for Al-tolerant and Alsensitive sorghum (Sorghum bicolour) genotypes grown with and without aluminium. Plant and Soil 134: 179-188.

Giaveno, G. D., Miranda Filho, J.B. and Furlani, P. R. 2001.Inheritance of aluminum tolerance in maize (Zea mays L.). Journal of Genetics and Breeding 55:51-56.

Hede, A.R., Skovmand, B., Ribaut, J.M., Gonzales-de-leon, D., Stolen O. 2002. Evaluation of aluminium tolerance in a spring rye collection by hydroponic screening. Plant Breeding 121(3): 241- 248.

Hill, P.R., Ahlrichs, J.L. and Ejeta, G. 1989. Rapid evaluation of sorghum for aluminum tolerance. Plant and Soil 114: 85 - 90.

International Sorghum and Millets Research/U.S Agency for International Development (INTSORMIL/USAID). 2006. The Atlas of Sorghum Production in Five Countries of
Eastern Africa. University of Nebraska, Lincoln.

Kochian, L.V., Hoekenga, O.A. and Piñeros, M.A. 2004. How do crop plants tolerate acid soils? Mechanisms of aluminum tolerance and phosphorous efficiency. Annual Review of Plant Biology 55: 459 - 493.

Konzak, C.F., Polle, E. and Kittrick, J.A, 1976. Screening several crops for aluminum tolerance. In: Wright, M.J and Ferrari, A.S. (Ed.). Proceeding of Workshop on Plant Adaptation to Mineral Stress in Problem Soils.

Ligeyo, D.O, 2007. Genetic analysis of maize (Zea mays L.) tolerance to aluminium toxicity and low phosphorus stress and development of synthetics for use in acid soils of Western Kenya. PhD thesis submitted in Department of Biological Science, Moi University, 2007.

Luo, H.M., Watanabe, T., Shimano, T. and Tadano, T. 1999. Comparison of aluminium tolerance and phosphate absorption between rape (Brassica napus L.) and tomato (Lycopersicum esculentum Mill.) in relation to organic acid exudation. Soil Science and Plant Nutrition 45: 897 - 907.

Ma, J.F., Hiradate, S. and Matsumoto, H. 1997 Specific secretion of citric acid induced by Al stress in Cassia tora L. Plant Cell Physiology 38:1019-1025.

Magalhães, J.V., Garvin, D.F., Wang, Y., Sorrells, M.E. et al. 2004. Comparative mapping of a major aluminum tolerance gene in sorghum and other species in the poaceae. Genetics 167:1905-1914.

Magalhaes, J.V., Caniato, F.F., Guimaraes, C.T., Schaffer, R.E., Alves, V.M.C., Borem, A., Klein, P.E. and Kochian, L.V.2007 Genetic diversity for Aluminium tolerance in sorghum. Theorerical and Applied Genetics 114: 863-876.

Magnavaca, R., Gardener, C.O. and Clark, R.B. 1987. Evaluation of inbred lines for aluminum tolerance in nutrient solution. In: Gabelman, H.W. and Loughman, B.C. (Eds.), pp. 255-265. Genetic aspects of plant mineral nutrition. Martinus Nijhoff Publ., Dordretcht, Netherlands. 
Mbwaga, A.M. 2006. Integrated Striga management to meet sorghum market demand in Tanzania. Striga conference, Purdue University 2006. Available at http:// www.agry.purdue.edu/strigaconference/pdf/ 23-Mbwaga.pdf

Mlingano Agricultural Research Institute (MARI). 2006. Rainfed agriculture crop sustainability for Tanzania: Technical Report. Ministry of Agriculture, Food and Co operatives, Tanga, Tanzania.

Nguyen, B.D., Brar, D.S., Bui, B.C. and Nguyen, T.V. 2003. Identification and mapping of the QTL for aluminum tolerance introgressed from the new source, Oryza rufipogon Griff., into indica rice (Oryza sativa L.). Theorerical and Applied Genetics 106: 583-593.

Paliwal, K., Sivaguru, M. and Thirselvi. 1994. Identification of Aluminium tolerant tropical cowpea cultivar growth and biomass accumulation parameters. Journal of Plant Nutrition 17:367 - 376

Rohrbach, D.D., Mtenga, K., Kiriwaggulu, J.A.B., Monyo, E.S., Mwaisela, F. and Saadan, H.M. 2002. Comparative study of three community seed supply strategies in Tanzania. International Crops Research
Institute for the Semi-Arid Tropics. Bulawayo, Zimbabwe.

SAS Version 8 System for Windows. 2002. The SAS Institute, U.S.A.

Tamas, L., Budikova, S., Simonovicova, M., Huttova, J., Siroka, B. and Mistrik, I. 2006. Rapid and Simple method for Al toxicity analysis in emerging barley roots during germination. Biologia Plantarum 50 (1):8793.

Villarcia, M.R., Carter, T.E., Rufty, T.W., Niewoehner, A.S., Jennette, M.W. and Arrellano, C. 2001. Genotypic rankings for aluminium tolerance of soybean roots grown in hydroponics and sand cultuire. Crop Science Journal 41:1499 - 1507.

Von Uexkûll, H.R. and Mutert, E. 1995. Global extent, development and economic impact of acid soils. pp 5-19. In: R.A. Date, N.J. Grundon, G.E. Raymet, M.E. Probert (eds), Plant-Soil Interactions at low $\mathrm{pH}$ : Principles and management. Kluwer Academic Publishers, Dordrecht, The Netherlands.

World Bank. 2005. Agricultural Growth for the poor: An Agenda for Development. The International Bank for Reconstruction and Development, The World Bank, Washington, D.C. 